\title{
Uso de Funções de Covariância na Descrição do Crescimento de Bovinos Nelore Criados no Estado de Pernambuco ${ }^{1}$
}

\author{
Kleber Régis Santoro², Severino Benone Paes Barbosa ${ }^{3}$, Eufrázio de Souza Santos ${ }^{4}$, Lúcia \\ Helena de Albuquerque Brasil ${ }^{5}$
}

\begin{abstract}
RESUMO - Este trabalho foi realizado com os objetivos de avaliar diferentes modelos de regressão aleatória, compostos por polinômios de Legendre, utilizados na descrição de efeitos genéticos e ambientais sobre observações do tipo peso-idade e identificar o mais adequado. Analisaram-se dados de peso-idade de bovinos Nelore, nascidos e criados no estado de Pernambuco, com pesagens ao nascimento e em intervalos de, aproximadamente, 90 dias até 720 dias de idade. Foram avaliados seis diferentes modelos de regressão aleatória, com comportamento de graus 3, 4 e 5 para os efeitos genético aditivo direto e de ambiente permanente, e dois tipos de comportamento para os erros (um homogêneo e outro heterogêneo com três classes). Utilizou-se o critério de informação de Akaike no julgamento do melhor modelo. O modelo mais adequado foi o de grau 5 com erros homogêneos. O comportamento predito pelo modelo para as correlações genéticas e fenotípicas foram baixos entre idades menores e maiores, altas e aproximadamente constantes para entre idades maiores. A covariância genética aditiva foi crescente com a idade. A herdabilidade esteve de baixa à média até aproximadamente 60 dias, sendo alta para as demais idades, ficando entre 0,50 e 0,60 .
\end{abstract}

Palavras-chave: critério de informação Akaike, polinômio de Legendre, regressão aleatória, teste de razão de verossimilhança

\section{Use of Covariance Functions to Describe the Growth Curve of Nelore Cattle, in Pernambuco State, Northeastern Brazil}

\begin{abstract}
Random regression models using Legendre polynomials were used to describe the growth curve of Nelore cattle weighted every three months from birth to 720 days of age, in Pernambuco state, northeastern Brazil. Six different random regression models using Legendre polynomials of three, four, and five degrees to model additive genetic and permanent environmental effects, under homogeneous and heterogeneous residual variances with three classes were evaluated. According to the Akaike's information criteria, the five degree Legendre polynomial with homogeneous error was the best fitting model. Genetic and phenotypic correlations were low between weights at early and late stages of the weighting period, and high and approximately constant between weights at late stages. Genetic covariance of weights increased with age. Heritability estimates were low to moderate up to 60 days of age and reached values between 0.50 and 0.60 at older ages.
\end{abstract}

Key Words: Akaike's information criteria, Legendre polinomial, likelihood ratio test, random regression

\section{Introdução}

As análises multivariadas ou univariadas geralmente empregam uma abordagem com modelos fatoriais ou em esquema de parcelas subdivididas na qual as diferentes observações são representadas pelas subparcelas (Littel et al., 1996; Johnson \& Wichern, 1998). Desta forma, cada uma das observações é considerada uma característica "diferente", isolada, produzindo um modelo de "janelas" ou "finito" (Kirkpatrick et al., 1990; Pletcher \& Geyer, 1999).
Outra abordagem em medidas repetidas, além do modelo finito, é a suposição de que a característica estaria mudando continuamente, em dimensão "infinita" (Kirkpatrick \& Heckman, 1989; Jaffrézic \& Pletcher, 2000). Entretanto, esse modelo "infinitesimal" (Kirkpatrick et al., 1990) impõe a necessidade de uma matriz de covariâncias de dimensão também "infinita". Uma solução encontrada para esta abordagem está na "função de covariância" (Henderson Jr., 1982; Longford, 1993), que especifica uma função apta a descrever o comportamento dos parâmetros da matriz de covariância em qualquer ponto desejado, inclusive

\footnotetext{
${ }_{1}^{1}$ Parte da tese de doutorado do primeiro autor financiada pelo CNPq.

2 Professor - UAG/UFRPE. Rua Ernesto Dourado, 82 - Bairro Heliópolis - CEP: 55296-190 - Garanhuns - PE. E.mail: ksantoro@ufrpe.br

3 Professor DZ/UFRPE. Rua Dom Manoel de Medeiros, s/n; Bairro de Dois Irmãos; 52171-900, Recife (PE). E.mail: sbarbosa@ufrpe.br

4 Professor Pós-Graduação em Biometria/UFRPE. Rua Dom Manoel de Medeiros, s/n; Bairro de Dois Irmãos; 52171-900, Recife (PE)

5 Professora DZ/UFRPE. Rua Dom Manoel de Medeiros, s/n; Bairro de Dois Irmãos; 52171-900, Recife (PE)
} 
naqueles não observados (Littell et al., 1996). As funções de covariância reduzem o número de parâmetros a serem estimados, diminuindo o esforço computacional e facilitando a compreensão dos resultados pelo pesquisador.

As funções de covariância estimadas por regressão aleatória têm sido largamente empregadas em dados de produção e crescimento animal (Kirkpatrick et al., 1990), com aplicações ao melhoramento genético animal (Meyer \& Hill, 1997; Meyer, 1998; Meyer, 1999; Meyer, 2000b).

Objetivou-se neste trabalho estudar diferentes modelos de regressão aleatória utilizados na descrição da variabilidade interindividual de dados de crescimento do tipo peso-idade de bovinos Nelore criados no estado de Pernambuco.

\section{Material e Métodos}

Foram selecionados, entre aqueles 14.035 animais Nelore com observações de peso-idade, aqueles que possuíam necessariamente nove pesagens, reduzindo a amostra para 925 animais, nascidos e criados no estado de Pernambuco. As pesagens ocorreram em intervalos de, aproximadamente, 90 dias, do nascimento aos dois anos de idade. Os animais eram filhos de 181 pais e 746 mães e seus registros constavam do banco de dados de controle de desenvolvimento ponderal da Associação Brasileira de Criadores de Zebu (ABCZ).

Foram criadas classes de idade de pesagem para cada dez dias de idade, reduzindo o número total de 712 diferentes idades para 73 classes etárias, não centradas (por exemplo, a classe 530 englobou animais de 530 a 539 dias de idade). Esse procedimento elevou o número de observações das idades simples para as classes formadas. As observações acima de 729 dias de idade foram desconsideradas por estarem disponíveis em número reduzido. Somente uma pesagem por animal foi permitida para cada classe de idade (a de menor idade entre todas da mesma classe), de modo que alguns animais tiveram o número de pesagens reduzido: quatro ficaram com sete registros; 188, com oito registros; e 733 , com nove registros, totalizando 8.129 observações de peso-idade.

Foi utilizado o seguinte modelo de regressão aleatória (Meyer, 1998; Kirkpatrick et al., 1990), no qual foram empregados os polinômios de Legendre:

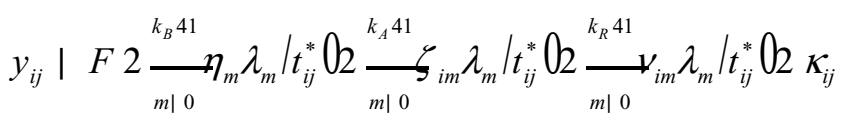

em que $y_{i j}$ é o peso do animal $i$ na idade $j ; F$, um conjunto de efeitos fixos; $t_{i j}^{*}$, a $j$-ésima idade para o animal $i$, padronizada para este intervalo; $I_{m} t_{i j}^{*} L, o$ $m$-ésimo polinômio de Legendre avaliado para $, b_{m}, \mathrm{o}$ $m$-ésimo coeficiente de regressão do peso sobre a idade (fixo); $a_{i m}$ e $g_{i m}$, o $m$-ésimo coeficiente de regressão aleatório para os efeitos genético aditivo direto e de ambiente permanente, respectivamente; e $e_{i j}$, o erro aleatório. As ordens dos polinômios a serem ajustados foram representadas por $k_{B}, k_{A}$ e $k_{R}$.

$\mathrm{O}$ termo $F$ do modelo incluiu os seguintes efeitos fixos: $\mu$ representando uma constante comum a todas as observações; $S_{k}$, o efeito do sexo $k$ ( $1=$ fêmea, $2=$ macho $) ; P_{n}$ representando o efeito da propriedade $n(n=1, \ldots, 41) ; T_{o}$, o efeito do regime de criação $o$ $(1=$ a pasto, $2=$ semi-estabulado, $3=$ estabulado, 4 = com mudança); $A_{l}$, o grupo contemporâneo de nascimento $l(l=1, \ldots, 39)$, representado pelo ano $($ ano $=1977, \ldots, 1997)$ e pela estação de nascimento (abril a setembro $=1=$ chuvosa, outubro a março $=$ 2 = seca).

Matricialmente, o modelo de regressão aleatória (Meyer, 1999) pode ser escrito como:

$$
\text { y } X b \square Z^{*} D \square Z_{D}^{*} J \square H
$$

em que $y$ é o vetor de $N$ observações medidas nos $N_{D}$ animais; $b$, o vetor de efeitos fixos; $a$, o vetor de $k_{A}$ x $N_{A}$ de coeficientes de regressão aleatória genética aditiva, no qual $N_{A}{ }^{3} N_{D}$ denota que o número total de animais em análise inclui progenitores sem registro; $\gamma$, o vetor de $k_{R} \times N_{D}$ de coeficientes de regressão aleatória para ambiente permanente; $\varepsilon$, o vetor de $N$ erros de medida; e $X, Z^{*}$ e são as matrizes de incidência dos efeitos.

Foram ajustados polinômios de Legendre de ordens 3,4 e 5 , igualmente para os efeitos genéticos aditivos direto, de ambiente permanente e de trajetória populacional. Assim, $k_{A}=3,4,5 ; k_{R}=3,4,5$ e $k_{B}=3$, 4,5 . O resíduo foi considerado independentemente de distribuído com medidas de variância homogêneas ou heterogêneas. Para as medidas heterogêneas, foram consideradas três classes de idade: 1 ( 1 até 240 dias), 2 ( 250 até 500 dias) e 3 (510 até 790 dias).

Foram analisados seis modelos de regressão aleatória (M331, M441, M551, M333, M443 e M553), em que M551 representou um modelo de ordem 5 para os polinômios de efeito genético aditivo direto $\left(k_{A}\right)$ e de ambiente permanente $\left(k_{R}\right)$ com medidas de erro homogêneas $(e)$. Realizaram-se, ainda, análises 
univariadas (Uni) para as idades médias das nove pesagens iniciais $(0,48,138,229,320,412,503,594 \mathrm{e}$ 686 dias) utilizando-se o software DXMRR versão 3.1 (Meyer, 2000a), considerando-se três diferentes pontos iniciais e convergência assumida a $10^{-6}$.

Os seguintes critérios de ajuste estavam disponíveis (Wolfinger, 1993):

a) $-2 l l=-2 \log$ verossimilhança:

b) critério de informação de Akaike (AIC) $=-2 l l+2 q$

c) critério bayesiano de Schwartz (BIC) $=-2 l l+q \log$ $(n-p)$

em que $q$ é o número de parâmetros de covariância; $n$, o número de observações; e $p$, o posto da matriz de incidência dos efeitos fixos no modelo, ou seja, da matriz $X$.

O modelo com melhor ajuste foi escolhido pelo menor valor de ajuste do critério de informação de Akaike (AIC).

\section{Resultados e Discussão}

O número de observações por classe de idade foi relativamente homogêneo (aproximadamente 100 registros por classe de idade), de modo que o desviopadrão para o peso aumentou com a idade, como esperado (Figura 1). O coeficiente de variação para o peso foi constante para as idades acima de 120 dias, com grande variação entre as idades inferiores, principalmente até os 60 dias (Figura 2).
Entre os critérios de seleção para o melhor modelo misto, encontram-se o teste assintótico da razão de verossimilhança, o critério de informação de Akaike (AIC) e o critério bayesiano de Schawrz (BIC). O primeiro, entretanto, só pode ser utilizado na comparação de dois modelos desde que uma matriz de covariância seja um caso especial da outra (Matsushita, 1994; Khattree \& Naik, 1999). O AIC pode ser emregado para comparar modelos com os mesmos efeitos fixos, mas diferentes estruturas de variância, sendo considerado melhor o modelo com o menor AIC. No caso do BIC, o julgamento é o mesmo, mas esse critério penaliza mais os modelos com grande número de parâmetros que o $\mathrm{AIC}$, de modo que os dois critérios podem não concordar (Wolfinger \& Chang, 1995; Xavier, 2000). Escolheu-se, então, a melhor matriz pelo critério AIC.

O critério de informação de Akaike demonstrou que o modelo mais adequado para a descrição dos dados foi o M551 (Tabela 1). Apesar de o modelo M553 estar próximo em valor AIC ao M551, esses modelos seriam estatisticamente diferentes segundo o teste de razão de verossimilhança (Tabela 2).

Os modelos apresentaram diferentes predições para o comportamento da herdabilidade (Figura 3). Esta diversidade também foi observada em outros trabalhos (Meyer, 2003a; Meyer \& Hill, 1997); entretanto, o comportamento geral encontrado neste estudo foi muito diversificado, especialmente do nascimento até próximo aos 100 dias, provavelmente em razão da grande variabilidade dos dados nessas idades (Figura 2).

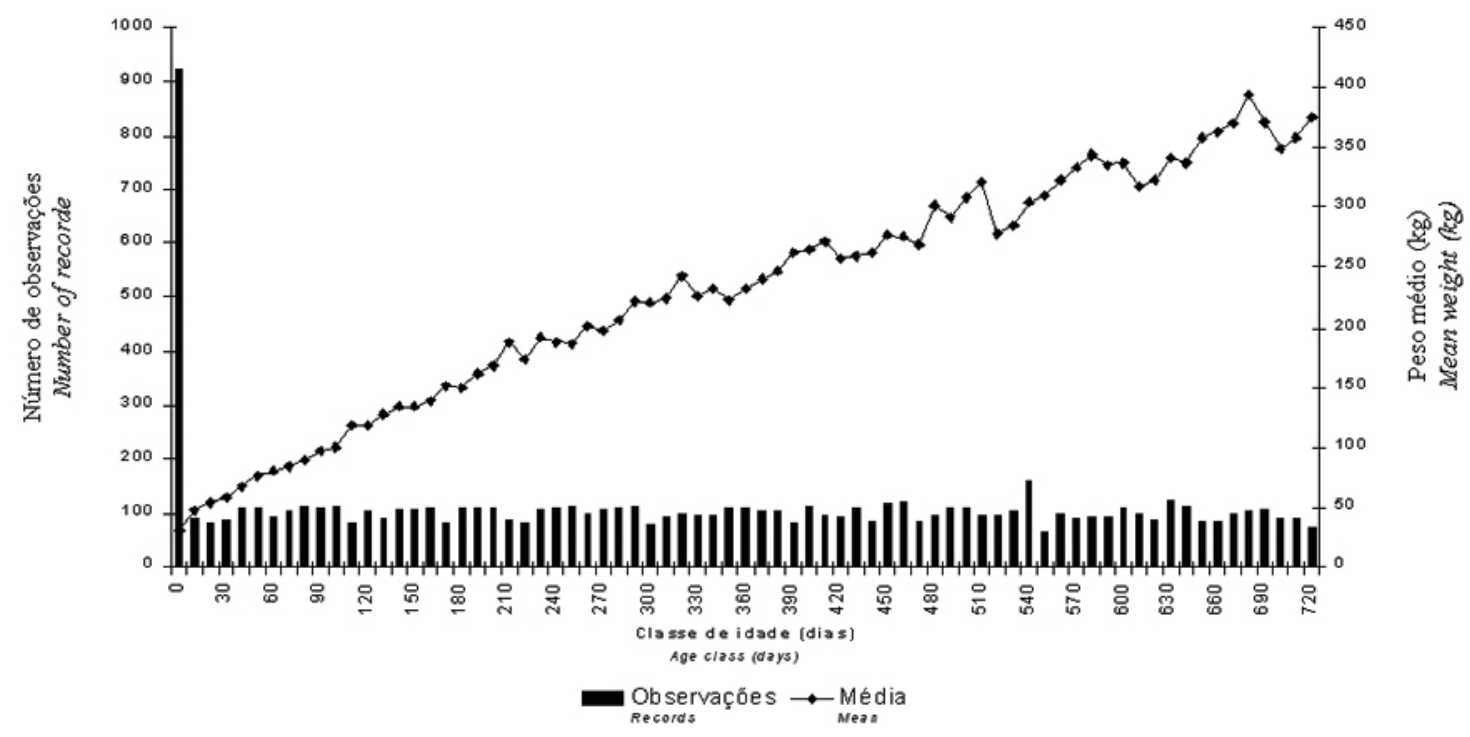

Figura 1 - Número de observações e peso médio $(\mathrm{kg})$ por classe de idade.

Figure 1 - Number of records and mean weight $(\mathrm{kg})$ by age class.

R. Bras. Zootec., v.34, n.6, p.2290-2297, 2005 (supl.) 
Tabela 1 - Ajuste obtido com o uso dos diferentes modelos utilizados

Table 1 - Obtained adjustment by different models

\begin{tabular}{lcccc}
\hline $\begin{array}{l}\text { Modelo } \\
\text { Model }\end{array}$ & $\begin{array}{c}\text { Parâmetro } \\
\text { Parameter }\end{array}$ & $\begin{array}{c}\text { Log } \\
\text { verossimilhança } \\
\text { Loglikelihood }\end{array}$ & $\begin{array}{c}\text { AIC } \\
\text { AIC }\end{array}$ & $\begin{array}{c}\text { BIC } \\
\text { BIC }\end{array}$ \\
\hline M331 & 13 & $-30701,52$ & 61429,04 & 61441,52 \\
M441 & 21 & $-30463,99$ & 60969,98 & 60990,06 \\
M551 & 31 & $-29907,20$ & 59876,40 & 59905,89 \\
M333 & 15 & $-30453,51$ & 60937,02 & 60951,41 \\
M443 & 23 & $-38902,46$ & 77850,92 & 77872,89 \\
M553 & 33 & $-29994,82$ & 60055,64 & 60087,00 \\
\hline
\end{tabular}

O comportamento delineado por dez diferentes modelos utilizados por Meyer (2003a) foi de herdabilidade média $(0,40)$ próximo ao nascimento, com decaimento rápido até baixa herdabilidade $(0,15)$, próximo aos 20 dias, crescimento rápido até média herdabilidade $(0,30$ a 0,40 ), próximo aos 150 dias, e, em seguida, aumento gradual até próximo aos 700 dias, alcançando herdabilidade média a alta $(0,40$ a 0,80$)$.

O comportamento da herdabilidade para o modelo M551 após os 90 dias foi relativamente constante, entre 0,50 e 0,60 . O declínio próximo aos 210 dias

Tabela 2 - Teste de razão de verossimilhança para os modelos estudados

Table 2 - Likelihood ratio test for the studied models

\begin{tabular}{|c|c|c|c|c|c|}
\hline \multirow[b]{2}{*}{$\begin{array}{l}\text { Modelo } \\
\text { Model }\end{array}$} & \multicolumn{5}{|c|}{$\begin{array}{c}\text { Modelo } \\
\text { Model }\end{array}$} \\
\hline & $\mathrm{M} 331^{\mathrm{a}}$ & M441 & M551 & M333 & M443 \\
\hline M441 & $475,06^{\mathrm{b}}(8)$ & & & & \\
\hline M551 & $1588,64(18)$ & $1113,58(10)$ & & & \\
\hline M333 & $496,02(2)$ & $20,96(6)$ & $1092,62(16)$ & & \\
\hline M443 & $16401,9(10)$ & $16876,94(2)$ & $17990,52(8)$ & $16897,9(8)$ & \\
\hline M553 & $1413,4(20)$ & $938,34(12)$ & $175,24(2)$ & $917,38(18)$ & $17815,28(10)$ \\
\hline
\end{tabular}

a Graus de liberdade entre parênteses, ${ }^{b}$ todos os valores são significativos a $1 \%(P<0,01)$.

a Degress of freedom between braces, " all values are significant at $1 \%(P<0.01)$.

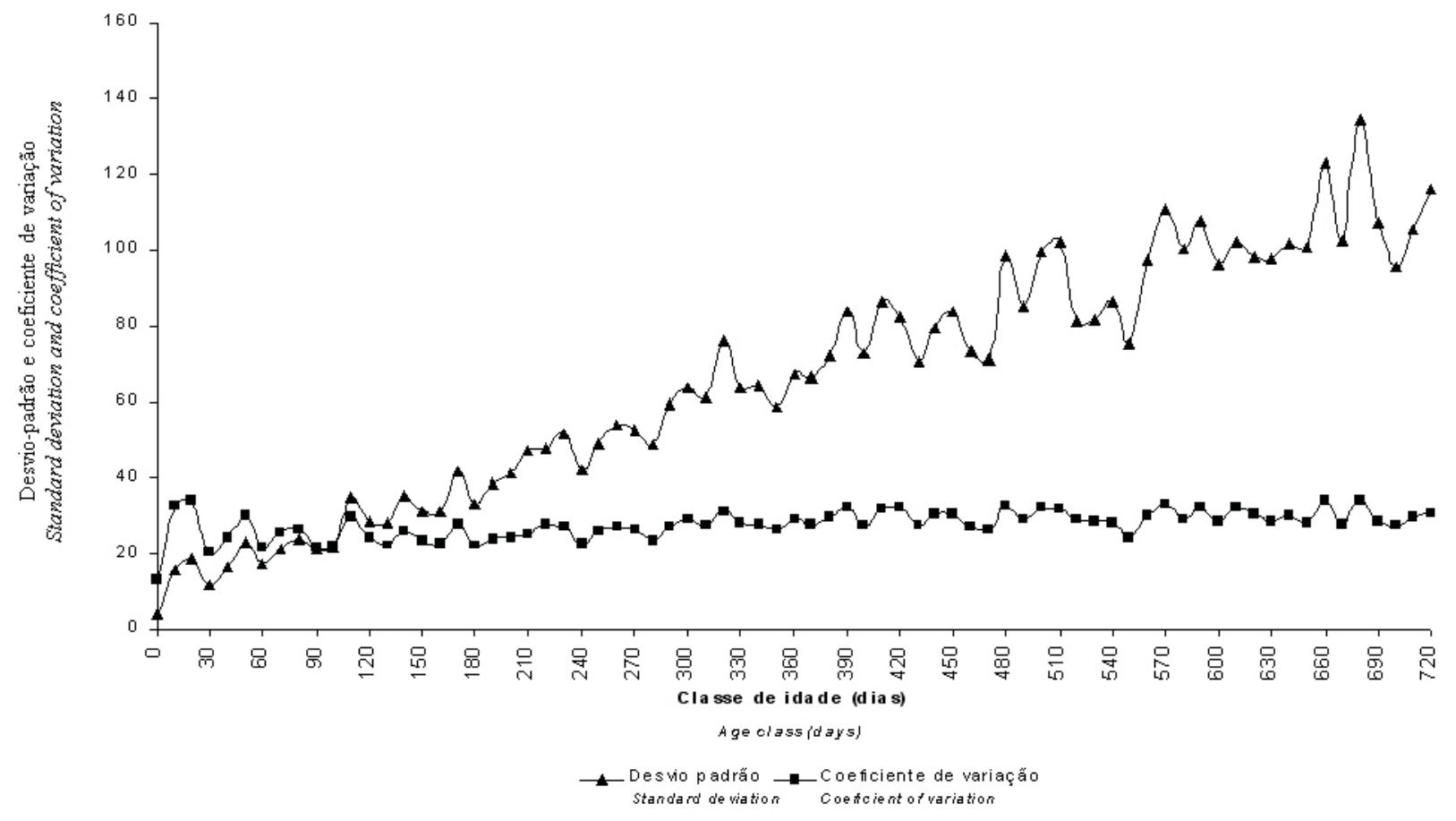

Figura 2 - Desvio-padrão e coeficiente de variação para a amostra.

Figure 2 - Standard deviation and coefficient of variation for the sample.

R. Bras. Zootec., v.34, n.6, p.2290-2297, 2005 (supl.) 
representaria o tempo médio de desmama dos animais, que geralmente ocorre aos 205 dias. Nesse ponto, ocorreria grande influência do meio sobre as características de crescimento, aumentando o efeito ambiental e diminuindo a herdabilidade, que começaria a elevar a partir de 540 dias, que é o período associado ao sobreano.

Vários fatores poderiam aumentar a adequação dos modelos aos dados, proporcionando melhores ajustes e predições para os componentes de variância. Entre eles, estaria o intervalo da classe de idades, que foi de dez dias, mas talvez devesse ser menor, como verificado por Albuquerque \& Meyer (2001), que utilizaram classes com quatro dias, e Meyer (2003a), que utilizou cinco dias, o que possivelmente permitiria análise mais sensível das diferenças entre idades.
Outro fator seria a inclusão de efeitos no modelo, como o materno, utilizado por Meyer (2003a), Lopes et al. (2002) e Albuquerque \& Meyer (2001). Tem-se ainda a exploração de maior número de combinações para os graus de ajuste dos polinômios, além dos estudados, embora Meyer (1999, 2003ab) tenha relatado que o uso de polinômios de alto grau poderia ocasionar dificuldades de convergência e estimativas irreais de variância para altas idades. Meyer (2003a) sugeriu que uma regressão quadrática para todos os efeitos aleatórios, representados pelo modelo M333, seria uma escolha conservadora e sensível. Entretanto, esse modelo não apresentou o melhor ajuste aos dados neste trabalho (Tabela 1).

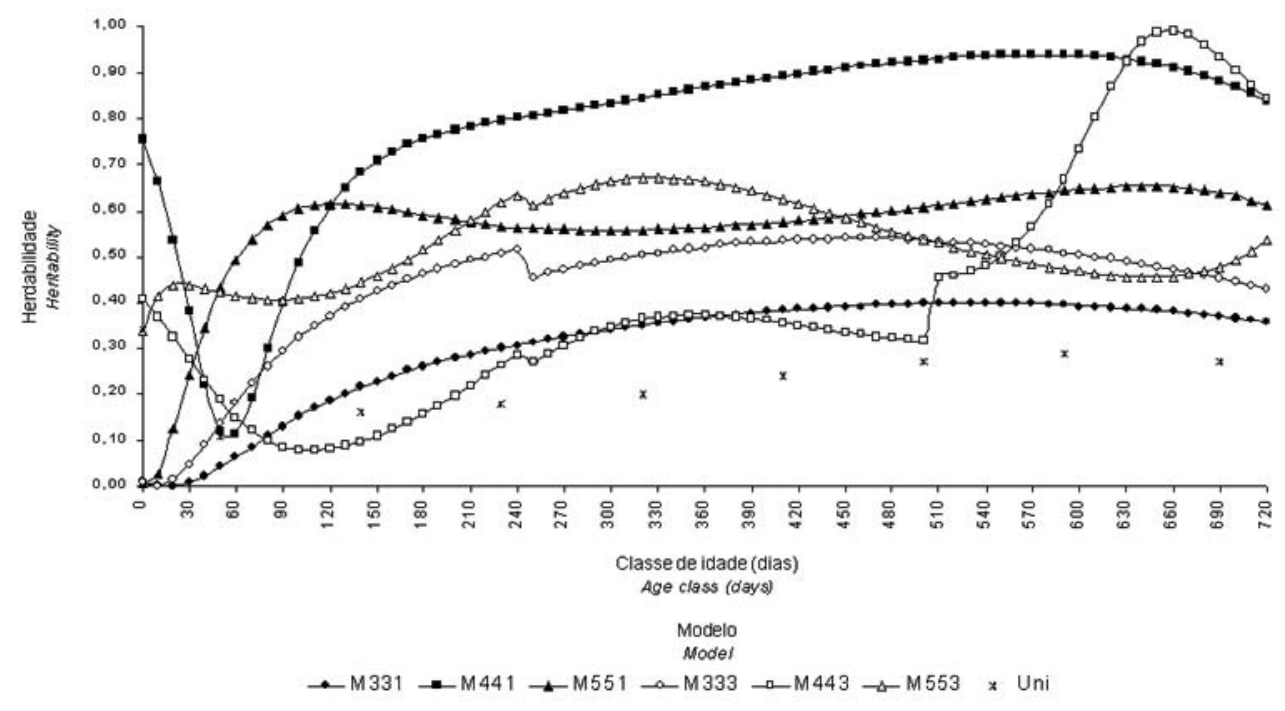

Figura 3 - Comportamento da herdabilidade de acordo com o modelo escolhido.

Figure 3 - Behavior of heritability in function of model.

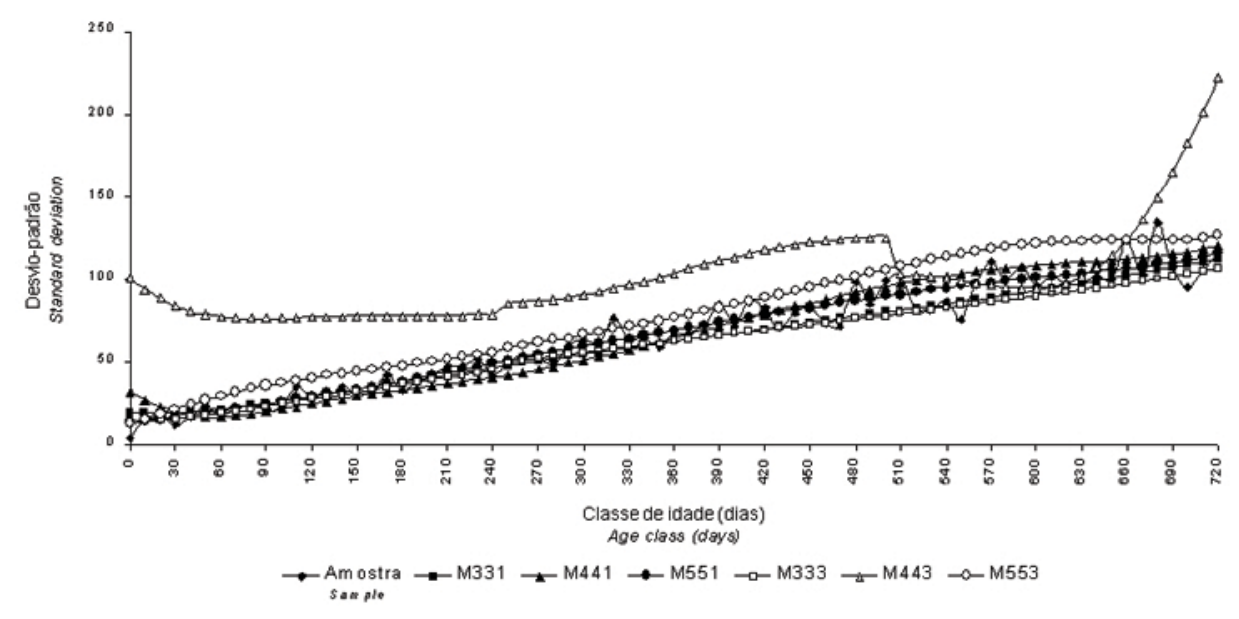

Figura 4 - Desvio-padrão na amostra e estimado pelos modelos.

Figure 4 - Standard deviation in sample and estimated by the models.

R. Bras. Zootec., v.34, n.6, p.2290-2297, 2005 (supl.) 
O tamanho da amostra também poderia ser considerado relativamente pequeno em relação aos descritos na literatura - Lopes et al. (2002): 71.867 e 74601; Meyer (2003a): 84.533; Albuquerque \& Meyer (2001): 20.065, 21.564 e 23.581; Meyer (2000b): 27.728 e 29.033 - mas esteve próximo ao estudado por Meyer \& Hill (1997), com 10.024 observações, e acima do reportado por Meyer (1999), com 3.320 e 3530 observações. Conforme salientado por Meyer (2003a), os resultados da análise por regressão aleatória seriam tão bons quanto os dados que a geraram. A influência da amostra nos resultados deste estudo poderia ser explicada pelo fato de que somente animais de relativo interesse reprodutivo seriam mantidos nos rebanhos até os 720 dias de idade ou mais, possuindo todas as nove pesagens, caso contrário, seriam comercializados. Além disso, estes animais também seriam melhor manejados que os demais animais do rebanho.

O desvio-padrão fenotípico predito pelos diferentes modelos, com exceção do M443, foi relativamente similar e o modelo que obteve predições mais próximas aos valores apresentados pela amostra foi o M551 (Figura 4). Estes resultados diferem dos descritos por Meyer (2000b), que encontrou respostas muito variadas entre polinômios de grau inferior a 6 e comportamento próximo a partir de polinômios de grau 12 .

A observação dos autovalores indicou maior peso para os dois primeiros autovalores, de forma que o primeiro autovalor ficou com o maior peso (Tabela 3), conforme reportado por Meyer (1998), Meyer (1999), Meyer (2003a) e Meyer \& Hill (1997). Segundo Meyer (1998), isso indica que a seleção feita para aumento de peso em qualquer das idades é o mesmo que elevar o peso em todas as demais idades.

Os resultados encontrados para a correlação genética aditiva direta foram compatíveis com os obtidos por Meyer (1999) e Meyer (1998) e apresentaram correlações baixas entre o peso nas idades iniciais e o peso nas idades finais (Figura 5). O comportamento da correlação de ambiente permanente também foi próximo ao verificado por Meyer (1999) (Figura 6), evidenciando correlação menor entre as diversas idades entre os 210 e 540 dias, aproximadamente. A correlação fenotípica demonstrou correlações baixas e até negativas entre pesos iniciais e finais, baixas correlações entre os pesos iniciais e altas correlações entre pesos de idades mais avançadas (Figura 7), corroborando os resultados registrados por Meyer (1999).

Há grandes vantagens no uso de modelos de regressão aleatória, podendo-se destacar o conhecimento do comportamento dos componentes de variância ao longo do tempo e de forma dinâmica, de modo que os resultados seriam ainda mais amplos e detalhados que em uma análise por modelos multivariados, não havendo a necessidade de se ter todos os registros de peso-idade, como necessário em uma regressão não-linear (Davidian \& Giltinan, 1995). Seria possível, portanto, trabalhar com animais que tivessem poucas observações ou observações incompletas, desde que estes animais não representassem a maior parte da amostra.

Entretanto, Meyer $(1999,2003 \mathrm{ab})$ recomendou cautela no emprego dos modelos de regressão aleatória, sobretudo em relação ao grau de ajuste dos polinômios, pois graus elevados poderiam resultar em estimativas irreais e problemas de convergência. Além disso, a escolha estaria também baseada nos recursos computacionais disponíveis, ressaltando-se ainda que não haveria um "roteiro" para a escolha do melhor modelo para uma aplicação em particular, uma vez que o melhor grau do polinômio, que poderia se basear em testes estatísticos para os autovalores, ainda não seria totalmente aceito, gerando controvérsias por não se conhecer corretamente sua distribuição.

Tabela 3 - Valores dos dois primeiros autovalores e soma dos autovalores para os modelos Table 3 - Values for the first two eigenvalues and sum of eigenvalues for the models

\begin{tabular}{lccccc}
\hline $\begin{array}{l}\text { Modelo } \\
\text { Model }\end{array}$ & $\lambda_{1}$ & $\lambda_{1} / \sum \lambda$ & $\lambda_{2}$ & $\lambda_{2} / \sum \lambda$ & $\sum \lambda$ \\
\hline M331 & 3408,390000 & 0,997471 & 8,640050 & 0,002529 & 3417,030067 \\
M441 & 9430,750000 & 0,940213 & 153,639000 & 0,015317 & 10030,437001 \\
M551 & 6257,710000 & 0,951862 & 249,015000 & 0,037878 & 6574,178283 \\
M333 & 4312,570000 & 0,996993 & 13,005100 & 0,003007 & 4325,575106 \\
M443 & 6389,650000 & 0,574487 & 0,013041 & 0,000001 & 11122,363041 \\
M553 & 6957,090000 & 0,902840 & 587,699000 & 0,076267 & 7705,782674 \\
\hline
\end{tabular}

R. Bras. Zootec., v.34, n.6, p.2290-2297, 2005 (supl.) 


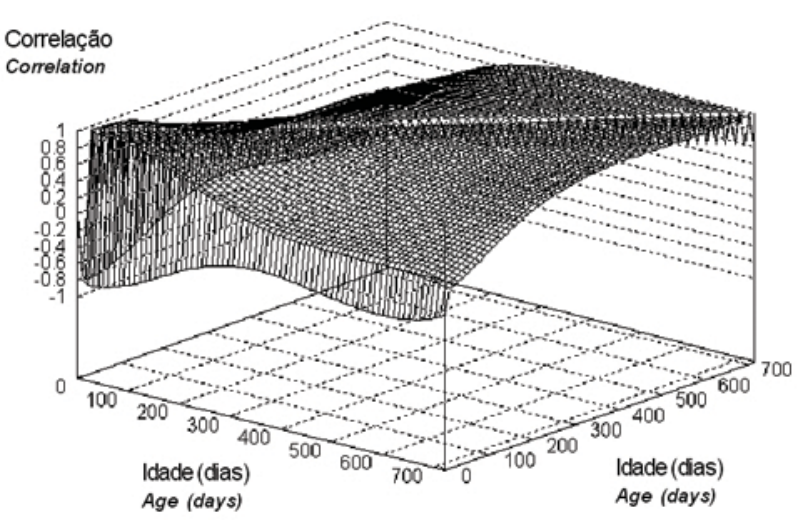

Figura 5 - Correlação genética aditiva direta entre as idades segundo o modelo M551.

Figure 5 - Genetic additive direct correlation between ages by M551 model.

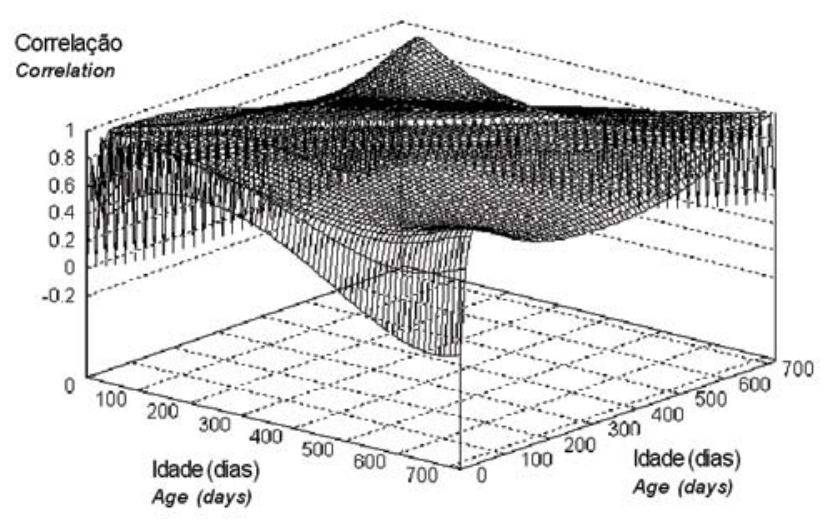

Figura 6 - Correlação de ambiente permanente entre as idades segundo o modelo M551.

Figure 6 - Environmental permanent correlation between ages by $M 551$ model.

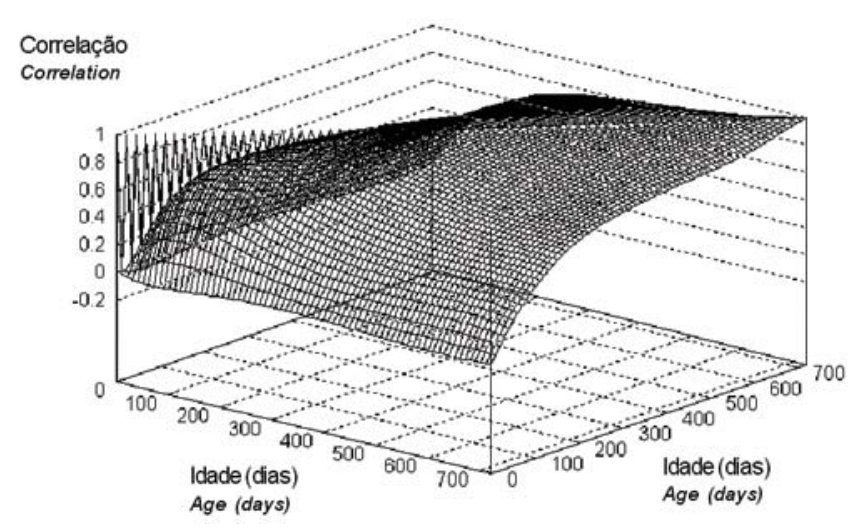

Figura 7 - Correlação fenotípica entre as idades segundo o modelo M551.

Figure 7 - Phenotypic correlation between ages by M551 model.

\section{Conclusões}

O modelo mais adequado para a descrição do comportamento dos componentes de variância genéticos e ambientais considera a distribuição de erros homogênea, juntamente a polinômios de Legendre de grau 5 para os efeitos genético direto, de ambiente permanente e trajetória média da população.

A partir dos modelos considerados na análise da amostra, foi possível um entendimento mais amplo do comportamento dos componentes de variância envolvidos. Entretanto, deve ser considerada a realização de uma nova análise com uma amostra maior e intervalos de classes de idade menores, na tentativa de se corrigir possíveis erros e melhorar os resultados obtidos.

$O$ uso de funções de covariância por meio de regressões aleatórias utilizando-se polinômios de Legendre na análise de medidas repetidas, apesar de grandemente atrativa, deve ser feita com cautela, pois ainda há dúvidas quanto ao correto grau de ajuste a ser aplicado ao polinômio e ao grande número de combinações possíveis entre os diversos efeitos considerados nos modelos.

\section{Literatura Citada}

ALBUQUERQUE, L.G.; MEYER, K. Estimates of covariance functions for growth from birth to 630 days of age in Nelore cattle. Journal of Animal Science, v.79, p.27762789, 2001.

DAVIDIAN, M.; GILTINAN, D.M. Nonlinear models for repeated measurement data. London: Chapman \& Hall, 1995. 359p.

HENDERSON JR., C.R. Analysis of covariance in a mixed model: higher-level, nonhomogeneous and random regression. Biometrics, v.38, p.633-640, 1982.

JAFFRÉZIC, F.; PLETCHER, S.D. Statistical models for estimating the genetic basis of repeated measures and other function-valued traits. Genetics, v.156, p.913922, 2000.

JOHNSON, R.A.; WICHERN, D.W. Applied multivariate statistical analysis. 4.ed. Englewood Cliffs: Prentice Hall, 1998. 532p.

KHATTREE, R.; NAIK, D.N. Applied multivariate statistics with SAS software. 2.ed. Cary: 1999. 338p.

KIRKPATRICK, M.; LOFSVOLD, D.; BULMER, M. Analysis of inheritance, selection and evolution of growth trajectories. Genetics, v.124, p.979-993, 1990.

KIRKPATRICK, M.; HECKMAN, N. A quantitative genetic model for growth, shape, and other infinite-dimensional characters. Journal of Mathematical Biology, v.27, p.429-450, 1989.

LITTELL, R.C.; MILLIKEN, G.A.; STROUP, W.W. et al. SAS System for mixed models. Cary: SAS Institute Incorporation, 1996. 633p. 
LONGFORD, N.T. Random coefficients models. Oxford: Clarendom Press, 1993. 390p.

LOPES, P.S.; NOBRE, P.R.C.; SILVA, L.O.C. et al. Análises de curves de crescimento de gado Nelore por RRM-REML. In: SIMPÓSIO NACIONAL DE MELHORAMENTO ANIMAL, 4., 2002, Campo Grande. Anais... Campo Grande: Sociedade Brasileira de Melhoramento Animal, 2002. p.119-121.

MATSUSHITA, R.Y. Modelos longitudinais mistos com correlação serial nos erros. Campinas: Universidade Estadual de Campinas, 1994. 188p. Dissertação (Mestrado em Estatística) - Universidade Estadual de Campinas, 1994.

MEYER, K. Estimating covariance functions for longitudinal data using a random regression model. Genetics, Selection, Evolution, v.30, p.221-240, 1998.

MEYER, K. Estimates of genetic and phenotypic covariance functions for postweaning growth and mature weight of beef cows. Journal of Animal Breeding and Genetics, v.116, p.181-205, 1999.

MEYER, K. DFREML - Version 3.0 b - user notes. Disnponível em $<$ http://agbu.une.edu.au/ kmeyer//dfreml.html $>$ Acessado em 13.07.2000. 29p. 2000a.

MEYER, K. Random regressions to model phenotypic variation in monthly weights of Australian beef cows. Livestock Production Science, v.65, p.19-38, 2000b.

MEYER, K. Estimates of genetic covariance functions for growth of Australian Angus cattle from random regression models fitting different orders of polynomials." In: ANNUAL MEETING OF THE EUROPEAN ASSOCIATION FOR ANIMAL PRODUCTION, 54., 2003, Roma. Proceedings... Roma: EAAP, 2003a. CDROM
MEYER, K. Random regression models for analyses of longitudinal data in animal breeding. In: Proceedings 54th Session of the International Statistical Institute, 54., 2003b, Berlin. Proceedings... Berlin: ISI, 2003b. CD-ROM

MEYER, K.; HILL, W.G. Estimation of genetic and phenotypic covariance functions for longitudinal data by restricted maximum likelihood. Livestock Production Science, v. 47, p. 185-200, 1997.

PLETCHER, D.; GEYER, C. J. The genetic analysis of agedependent traits: modeling the character process. Genetics, v. 151, p. 825-835, 1999.

WOLFINGER, R. Covariance structure selection in general mixed models. Communications in Statistics - Simulation, v.22, n.4, p.1079-1106, 1993.

WOLFINGER, R.; CHANG, M. Comparing the SAS GLM and MIXED procedures for repeated measures. In: ANNUAL SAS USERS GROUP CONFERENCE, 20., 1995, Cary (NC). Proceedings... Cary: SUG, 1995. CD-ROM.

XAVIER, L.H. Modelos univariado e multivariado para análise de medidas repetidas e verificação da acurácia do modelo univariado por meio de simulação. Piracicaba: Escola Superior de Agricultura "Luiz de Queiroz", 2000.91p. Dissertação (Mestrado em Estatística Experimental) - Escola Superior de Agricultura "Luiz de Queiroz”, 2000.

Recebido em: 13/04/04

Aceito em: 15/07/05 